\title{
Voice characteristics in adults with neurofibromatosis type 1
}

\author{
Marjan Cosyns ${ }^{1}$, Geert Mortier ${ }^{2}$, Paul Corthals ${ }^{1}$, Sandra Janssens ${ }^{2}$, and John Van Borsel ${ }^{1}$
}

${ }^{1}$ Department of Oto-rhino-laryngology and Logopaedic-Audiologic Sciences, Ghent University, Belgium

${ }^{2}$ Center for Medical Genetics, Ghent University Hospital, Belgium

Introduction: Several authors have mentioned the occurrence of voice abnormalities in patients with neurofibromatosis type 1 (NF1). However, most studies relied on perceptual evaluations only. To the best of our knowledge, no studies examined the voice characteristics of NF1 patients objectively by means of a multiparameter approach.

Purpose: $\quad$ Therefore, the aim of the present study was to document the voice characteristics in adult NF1 patients objectively in terms of aerodynamic measurements, voice range profiles, acoustic voice quality and intonation measurements, and dysphonia severity indices.

Method

The participants of this study involved a patient group and a control group.

- Patient group: 22 adults with NF1:

- 9 males (18- 64 y, mean 39;4 y)

- 13 females (17 - 48 y; mean 32;8 y)

- Exclusion criteria: laryngeal or pharyngeal neurofibroma smoking

The following voice assessment techniques were used:

- Voice range profile:

- Minimal and maximal pitch $\left(F_{\text {low }}\right.$ and $\left.F_{\text {high }}\right)$

- Minimal and maximal intensity $\left(\mathrm{I}_{\text {low }}\right.$ and $\left.\mathrm{I}_{\text {high }}\right)$

- Acoustic analysis:

$\begin{aligned} & \text { - Jitter } \\ & \text { - Shimmer } \\ & \text { - Noise-to-harmonic ratio (NHR) } \\ & \text { - Mean fundamental frequency }\left(F_{0}\right) \\ & \text { - Standard deviation of } F_{0}(S T D) \\ & \text { - Pitch variation }\end{aligned}$ - reading

- Aerodynamic measurements:

- Maximum phonation time (MPT)

- Vital capacity (VC)

- Phonation quotient (PQ)

- Dysphonia Severity Index (DSI)
- Control group: 22 healthy adults:

- 12 males (18 - $67 \mathrm{y}$, mean 38;0 y)

- 10 females (22 - 43 y, mean 32;11 y)

- Exclusion criteria: voice problem within 5 years smoking

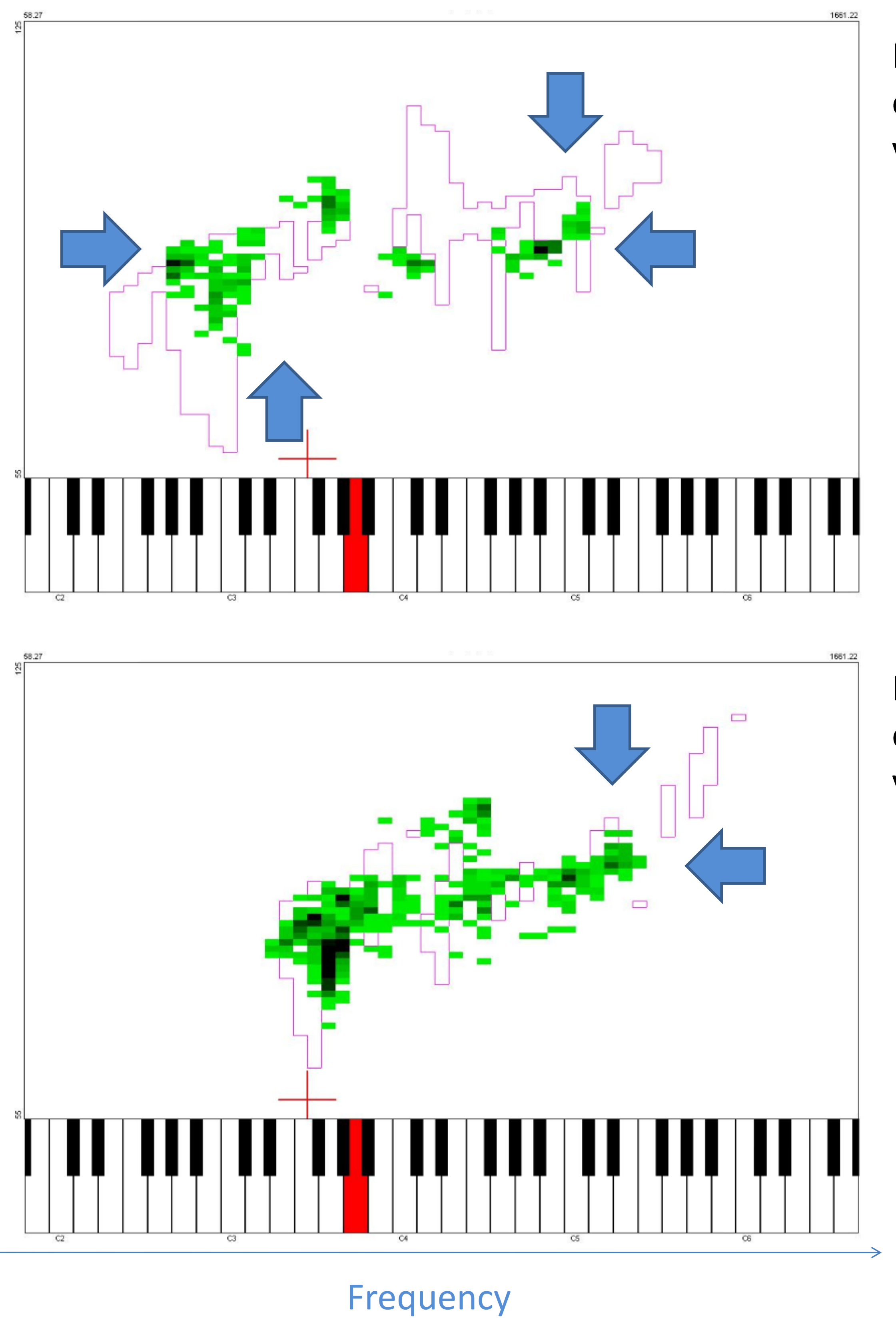

Figure 1: Voice range profile of an adult male with NF1 versus a healthy male.

Figure 2: Voice range profile of an adult female with NF1 versus a healthy female.

\section{Results}

- Voice range profile:

Males: $\quad \circ F_{\text {high }} \downarrow(p=0.015), F_{\text {low }} \uparrow(p=0.053)$ and $F_{\text {range }} \downarrow(p=0.011)$

$\circ I_{\text {high }} \downarrow(p=0.027), I_{\text {low }} \uparrow(p=0.001)$ and $I_{\text {range }} \downarrow(p<0.001)$

Females: $\circ F_{\text {high }} \downarrow(p<0.001)$ and $F_{\text {range }} \downarrow(p<0.001)$

。 $I_{\text {high }} \downarrow(p=0.002)$ and $I_{\text {range }} \downarrow(p=0.010)$
- Acoustic analysis:

- STD $\downarrow$ (males $p=0.059$, females $p=0.019$ )

- Aerodynamic measurements:

- VC $\downarrow$ (males $p=0.042$, females $p=0.004$ )

- DSI $\downarrow$ (males $p=0.006$, females $p=0.001$ )

\section{Discussion}

The results indicate that the overall voice quality is worse in NF1 patients compared to controls. Particularly, a decreased vital capacity and limitations in laryngeal possibilities with respect to both frequency and intensity were observed. Generally, respiration in function of phonation seems to be sufficient and hoarseness appears to be as prevalent in NF1 patients as in controls. Further, it seems that NF1 patients do intonate, but that the extent of their pitch variations is not as large as in controls.

The etiology of these voice deviations has yet to be established. We would like to put forward two alternative hypotheses. Paraspinal nerve root neurofibromas or neurofibromas occurring along the cervical plexus, which are commonly reported locations of neurofibromas in NF1, could interfere with the innervation of the respiratory muscles and give cause to the voice abnormalities observed. On the other hand, some authors suggested a neurological basis for the speech problems in NF1 patients. As a reduction in vital capacity and a decreased frequency variability can also be found in patients with Parkinson disease, the results of the present study remind of extrapyramidal involvement. Further research to clarify these issues is needed. 